\title{
Ecosystemic Services Linked to Water and Plant diversity of the San José de Chazo-Santa Fe de Galán Region
}

\section{Servicios Ecosistémicos Ligados al Agua y Diversidad Florística en el Páramo de la Ciénega en la Regional San José de Chazo- Santa Fe de Galán}

Memories II International Congress Forests and Agroforestry for the 21st Century

Corresponding Author:

J. Murillo Conterón

Published: 21 January 2021

Production and Hosting by Knowledge E

(c) J. Murillo Conterón et al. This article is distributed under the terms of the Creative Commons Attribution License, which permits unrestricted use and redistribution provided that the original author and source are credited.

\section{J. Murillo Conterón, R. Peña Murillo, and D. Román Robalino}

Facultad de Recursos Naturales, Escuela Superior Politécnica de Chimborazo, Riobamba, Ecuador

\section{Abstract}

In the higher part of the Santa Fe de Galán parish, intensive agricultural activities are carried out that cause problems of erosion, disappearance of species of fauna and flora, decrease in water and deterioration of páramo areas due to the advance of the agricultural frontier. These activities present a threat to the sustainability of the San José de Chazo-Santa Fe de Galán region, due to the degradation of its natural resources that directly affect the vital development of 1200 families belonging to this region. The present investigation aims to study ecosystem services linked to water, as well as floristic diversity, for which data was collected and systematized from the Ciénega páramo in the San José de Chazo-Santa Fe de Galán region. According to the data collected on floristic diversity, 29 samples of terrestrial vascular plants were registered, corresponding to 18 families, 26 genera and 29 species; moss and lichen of unidentified family, genus and species were also collected. The highest index of importance value by species and family was presented by the moss with $20.39 \%$ and $18.17 \%$, respectively. The average monthly flow during the February-May period observed through the volumetric method was $6.34 \mathrm{~L} / \mathrm{s}$.

Keywords: capacity, flow, diversity, paramo, ecosystem services, sustainability.

\section{Resumen}

En la parte alta de la Parroquia Santa Fe de Galán se desarrollan actividades agropecuarias intensivas que provocan problemas de erosión, desaparición de especies de fauna y flora, disminución del agua y deterioro de áreas de páramo por el avance de la frontera agrícola. Estas actividades representan una amenaza para la sostenibilidad de la Regional San José de ChazoSanta Fe de Galán, debido a la degradación de sus recursos naturales que inciden directamente en el desarrollo vital de 1200 familias pertenecientes a esta Regional. La presente investigación tiene como objetivo estudiar los servicios ecosistémicos ligados al agua y diversidad florística, cuyos datos fueron recolectados y sistematizados del páramo de la Ciénega en la Regional San José de Chazo-Santa Fe de Galán. De acuerdo con los resultados de diversidad florística se registró 29 muestras de plantas vasculares terrestres, correspondiente a 18 familias, 26 géneros y 29 especies, también se recolectó musgo y liquen de familia, género y especie no identificados. El mayor índice de valor de importancia por especie y familia lo presentó el musgo con $20,39 \%$ y $18,17 \%$, respectivamente. El caudal promedio mensual durante el período febrero - mayo a través del método volumétrico fue de 6,34 L/s. 
Palabras Clave: aforo, caudal, diversidad, páramo, servicios ecosistémicos, sostenibilidad.

\section{Introducción}

Los páramos tienen una importancia fundamental para millones de personas y representan una multiplicidad de significados y valores: Hábitats en los que sobreviven especies endémicas, ecosistemas capaces de brindar servicios ambientales fundamentales, espacios de importancia cultural, entre otros [1]. La biodiversidad de los páramos tiene valores impresionantes, aparte de su alto grado de endemismo, un aspecto interesante de su flora son las adaptaciones morfológicas de las plantas al ambiente. La regulación hídrica es el valor más notable de los páramos. Esta importancia se debe a un balance hídrico positivo, a la neblina que cubre grandes extensiones durante la mayoría del tiempo, a la estructura de la vegetación que capta el agua, la conduce al suelo y a su vez lo protege contra erosión y disecación, y al suelo humífero. Se puede decir que cada metro cuadrado de páramo 'produce' $1 \mathrm{~L}$ de agua por día [2]. El desconocimiento de los seres humanos con respecto a los servicios ecosistémicos que nos brinda el páramo ha hecho que las actividades económicas como los cultivos agrícolas, la ganadería, las plantaciones forestales, la minería, el manejo inadecuado del agua y la expansión urbana se conviertan en riesgos severos para la integridad de los páramos [1]. Además el ecosistema páramo no está evolutivamente adaptado a la presencia y acción de grandes herbívoros. Aparte de que en muchos casos arrancan de raíz las plantas, el propio peso de los individuos y la forma de sus cascos generan cambios irreversibles en la vegetación y el suelo $[3,4]$. Este impacto, aparte de los daños inmediatos y mediatos sobre la biodiversidad y el ambiente en términos amplios, se manifiesta en un descenso en la calidad de vida tanto de la gente que vive directamente del ecosistema, como de la que vive indirectamente del páramo [5].

En la parte alta de la parroquia Santa Fe de Galán se desarrollan actividades agropecuarias intensivas que provocan problemas de erosión, desaparición de especies de fauna y flora, disminución del agua y deterioro de áreas de páramo por el avance de la frontera agrícola. Estas actividades representan una amenaza para la sostenibilidad de la Regional San José de Chazo - Santa Fe de Galán, debido a la degradación del recurso edafológico, florístico y a la disminución de caudales hídricos que inciden directamente en el desarrollo vital de 1200 familias pertenecientes a esta Regional. Es por esta razón el interés de realizar el presente trabajo de investigación sobre el páramo y los servicios ecosistémicos ligados al agua y diversidad florística que este brinda. 


\section{Materiales y Métodos}

\section{1. Área de studio}

El estudio se realizó en el Páramo de la Ciénega mismo que representa una zona de recarga hídrica para la Regional, se encuentra ubicada en una altitud que va de los 3300 m.s.n.m. a 4200 m.s.n.m., aproximadamente. La regional tiene una superficie aproximada de 309,5 hectáreas. La zona se caracteriza por tener la presencia de neblina, cuya precipitación media es de $488,97 \mathrm{~mm}$; la temperatura media es de $11^{\circ} \mathrm{C}$ [6]. Además se distinguieron 3 zonas de vida: Arbustal siempre verde y herbazal del Páramo; herbazal inundable del Páramo y herbazal del Páramo según la clasificación del MAE [7]. La red fluvial forma parte de la cuenca del río Pastaza, subcuenca del río Chambo [6]. Es importante mencionar que aún existen áreas de tipo privado que son utilizados para la crianza de ganado bravo. Se realizó 20 sub-parcelas de $1 \mathrm{~m}^{2}$ (Tabla 1) y 3 zonas de aforo (Tabla 2; Figura 1).

\section{Table 1}

Ubicación de las sub-parcelas de muestreo en la regional.

\begin{tabular}{|c|c|c|c|}
\hline Punto & $\mathbf{S}$ & w & Altura \\
\hline P11 & 763458 & 9833692 & 3627 m \\
\hline P1 2 & 763458 & 9833688 & $3623 \mathrm{~m}$ \\
\hline P1 3 & 763452 & 9833691 & $3622 \mathrm{~m}$ \\
\hline P1 4 & 763449 & 9833698 & $3627 \mathrm{~m}$ \\
\hline P2 1 & 763060 & 9833209 & $3604 \mathrm{~m}$ \\
\hline P2 2 & 763072 & 9833212 & $3605 \mathrm{~m}$ \\
\hline P2 3 & 763069 & 9833199 & $3608 \mathrm{~m}$ \\
\hline P2 4 & 763056 & 9833201 & 3607 m \\
\hline P3 1 & 763275 & 9832750 & $3568 \mathrm{~m}$ \\
\hline P3 2 & 763273 & 9832746 & 3569 m \\
\hline P3 3 & 763278 & 9832740 & $3569 \mathrm{~m}$ \\
\hline P3 4 & 763285 & 9832744 & $3568 \mathrm{~m}$ \\
\hline P4 1 & 763602 & 9832732 & $3485 \mathrm{~m}$ \\
\hline P4 2 & 763605 & 9832736 & $3485 \mathrm{~m}$ \\
\hline P4 3 & 763608 & 9832740 & $3483 \mathrm{~m}$ \\
\hline P4 4 & 763606 & 9832733 & 3484 m \\
\hline P5 1 & 763825 & 9832866 & 3831 m \\
\hline P5 2 & 763822 & 9832866 & 3857 m \\
\hline P5 3 & 763819 & 9832862 & 3857 m \\
\hline P5 4 & 763823 & 9832859 & $3856 \mathrm{~m}$ \\
\hline
\end{tabular}


Table 2

Ubicación de las zonas de aforo en la regional.

\begin{tabular}{ll|l|l|l} 
Zona & Descripción & S & W & Altura \\
Zona 1 & Vertiente & 763956 & 9833112 & $3807 \mathrm{~m}$ \\
Zona 2 & Reservorio 1 & 763811 & 9833076 & $3845 \mathrm{~m}$ \\
\hline Zona 3 & $\begin{array}{l}\text { Reservorio de } \\
\text { cloración }\end{array}$ & 768249 & 9833547 & $3801 \mathrm{~m}$
\end{tabular}

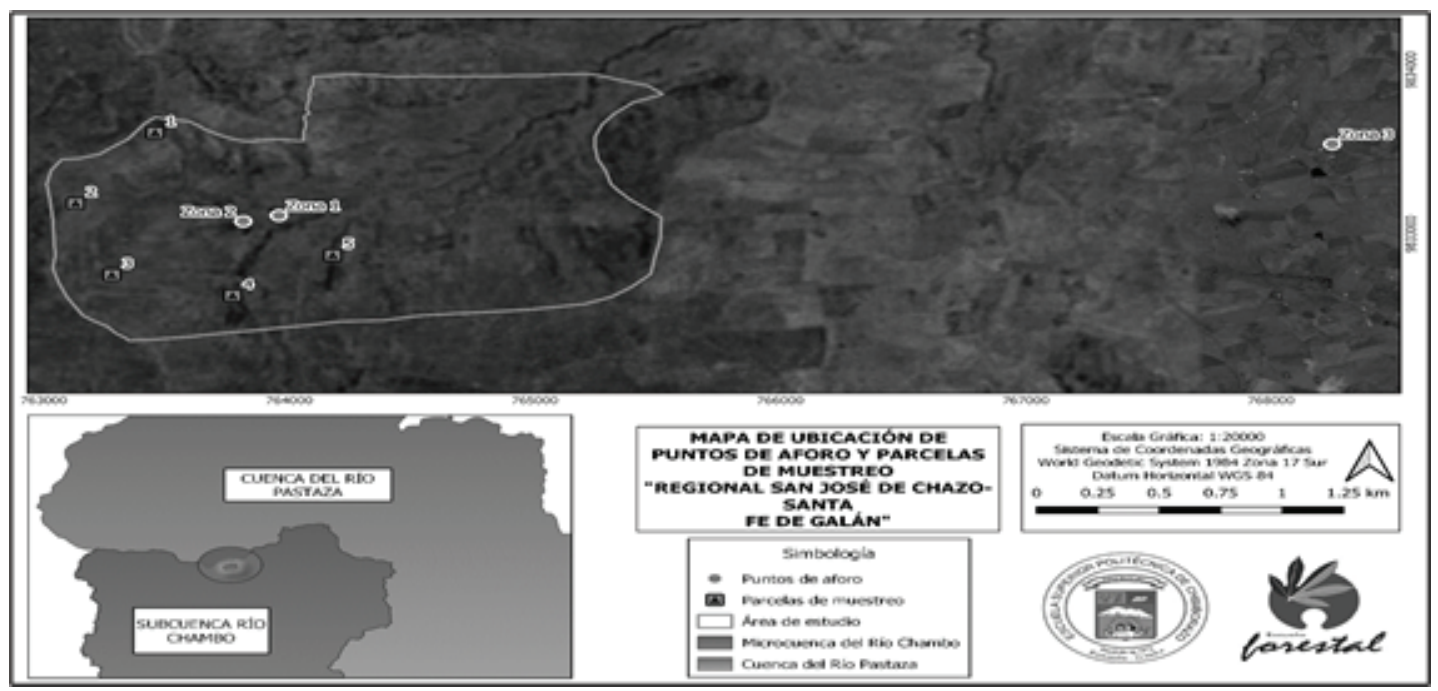

Figure 1

Ubicación de las parcelas de muestreo y zonas de aforo en la Regional San José de Chazo-Santa Fe de Galán.

\subsection{Diseño de muestreo}

\subsubsection{Diversidad florística}

La metodología utilizada para la instalación de las parcelas de muestreo fue la propuesta por Pauli et al. [8], para el Proyecto Gloria tomando en cuenta algunas modificaciones para adaptar a los páramos andinos. Se instalaron 5 parcelas de muestreo de $5 \times 5 \mathrm{~m}$, ubicadas a $300 \mathrm{~m}$ de distancia y en distintos lugares tomando en cuenta la pendiente, accesibilidad y el estado de cada sitio. Cada parcela de muestreo fue dividida en subparcelas de 1×1 m, las observaciones y recolección de vegetación se llevó a cabo únicamente en las sub-parcelas de las esquinas ya que las otras se alteraron con el pisoteo mientras se realizaba la recolección de las muestras, obteniéndose el registro de 20 sub-parcelas de $1 \times 1 \mathrm{~m}$. Los sub-cuadrantes de $1 \times 1 \mathrm{~m}$, se sub-dividieron en celdillas de 0,1 $\times 0,1 \mathrm{~m}$, para lo cual se utilizó un armazón de madera con un enrejado de hilos que delimitaron un total de 100 celdillas.

Las especies vegetales que se recolectaron en la zona de estudio fueron herborizadas en el mismo lugar, las especies fueron secadas manualmente bajo sombra, se cambió de papel periódicamente para evitar la putrefacción de las muestras o que 
estas se llenen de hongos. Dentro de las parcelas se registró número de individuos, frecuencia y cobertura de cada especie, con el fin de obtener datos cuantitativos de la vegetación, las especies que no se pudieron identificar en el campo fueron registradas con códigos para su posterior identificación en el herbario de la ESPOCH.

\subsubsection{Caudal}

Se aplicó el método volumétrico propuesto por Peralta [9], para el proyecto de titulación. El aforo consistió en llenar un balde de $10 \mathrm{~L}$, registrando el tiempo en que tarda en Ilenarse, efectuándose 3 repeticiones en el mes y por zona de aforo: Zona 1 (Vertiente), Zona 2 (Reservorio 1) y Zona 3 (Reservorio de cloración).

\subsection{Análisis de datos}

\subsubsection{Diversidad florística}

Se realizó un listado de las especies con sus respectivos datos con los cuales se obtuvo: índice de valor de importancia (IVI) por especie y familia, índice de Shannon-Weaver, índice de Simpson e índice de Sorensen, calculados en el software de office Excel.

Estos índices corresponden a una medida de la heterogeneidad de una comunidad en función de la riqueza y la abundancia de las especies. La diversidad permite distinguir entre dos comunidades con idéntica riqueza y composición florística, en la cual las especies difieren en cuanto a su abundancia relativa [10].

Según Campo y Duval [11], el índice de valor de importancia define cuáles de las especies presentes contribuyen en el carácter y estructura de un ecosistema. Este valor se obtiene mediante la sumatoria de la frecuencia relativa, la densidad relativa y la dominancia relativa.

Para tener idea de (número de individuos de una especie con relación al total de individuos de la población) [12] citado por [13]. La densidad relativa se refiere al porcentaje con el que cada especie contribuye al conjunto de la comunidad [14] citado por [13].

$$
\mathrm{Dr}=\frac{\text { total de individuos especie } \mathrm{A}}{\text { total de individuos, todas las especies }} \times 100
$$

Frecuencia relativa (FR).

$$
\text { Frecuencia Relativa }=\frac{\text { valor de frecuencia de la especie } A}{\text { valor total de frecuencia, todas las especies }} \times 100
$$

Dominancia relativa.

$$
\text { Dominancia relativa }=\frac{\text { área cobertura } \mathrm{sp} \mathrm{A}}{\text { área de cobertura de todas las } \mathrm{sp}} \times 100
$$


El Índice de diversidad de Shannon-Weaver es un índice basado en la equidad, expresa la uniformidad de los valores de importancia através de todas las especies de la muestra (Valencia, 2013). Los valores se interpretaron de acuerdo a la Tabla 3.

$$
H^{\prime}=-\sum_{i=1}^{S} p i\left(\operatorname{In} p_{i}\right),
$$

dónde $H^{\prime}$ es el Índice de Shannon-Weaver; $\mathrm{S}$ es el número de especies; $p_{i}$ es la proporción del número total de individuos que constituyen la especie.

El Índice de diversidad de Simpson está basado en la dominancia, son parámetros inversas al concepto de uniformidad o equidad de la comunidad. Tomando en cuenta la representatividad de las especies con mayor valor de importancia sin evaluar la contribución del resto de las especies [15]. Los valores se interpretaron de acuerdo a la Tabla 3.

$$
I S D=1-\sum(p i)^{2},
$$

dónde ISD es el Índice de Simpson; pi es la proporción del número total de individuos que constituyen la especie.

\section{Table 3}

Interpretación del Índice de Shannon y Simpson.

Valores
$0,00-0,35$
$0,36-0,75$
$0,76-1,00$

\section{Interpretación}

Diversidad baja

Diversidad mediana

Diversidad alta

Fuente: Referencias [16, 17].

El Índice de diversidad de Sorensen no considera la abundancia relativa de las especies. Es mucho más útil cuando el principal interés es la determinación de la presencia o ausencia de las especies [14] citado por [13]. Los valores se interpretaron de acuerdo a la Tabla 4.

$$
I s s=\frac{2 C}{A+B} \times 100,
$$

dónde Iss es el Índice de Sorensen; $A$ es el número de especies en el sitio 1; $B$ es el número de especies en el sitio 2 ; $C$ es el número de especies similares presentes en ambos sitios $A$ y $B$.

\subsubsection{Caudal}

El caudal se entiende que es el volumen de agua que traspasa una superficie en una unidad de tiempo [18].

El método Aforo volumétrico permite medir pequeños caudales de agua. Para ello es necesario contar con un balde de volumen conocido en el cual se colecta el agua, anotando el tiempo que demora en llenarse. Esta operación puede repetirse 2 o 3 


\section{Table 4}

Interpretación del Índice de Sorensen.

Valores
$0,00-0,35$
$0,36-0,70$
$0,71-1,00$

\section{Interpretación}

Disimiles

Medianamente similares

Muy similares

Fuente: Referncias $[13,16]$.

veces y se promedia con el fin de asegurar una mayor exactitud, aplicando la siguiente formula [19].

$$
Q=\frac{V}{T},
$$

dónde $\boldsymbol{Q}$ es el caudal en $\left(\mathrm{m}^{3} / \mathrm{s}\right) ; V$ es el volumen en $\left(\mathrm{m}^{3}\right)$ y $T$ es el tiempo en (s).

En Ecuador, la legislación ambiental comenzó a exigir la definición de un caudal ecológico, la norma establecía que se tome como referencia al menos el 10\% del caudal medio anual para el cálculo del caudal ecológico. El caudal ecológico debería permitir el funcionamiento y mantenimiento de los ecosistemas acuáticos [20].

\section{Resultados y Discusión}

\subsection{Riqueza florística}

Se recolectó 29 muestras de plantas vasculares terrestres, correspondiendo a 18 familias botánicas, 26 géneros y 29 especies, también se recolectó 1 musgo y 1 liquen de familia, género y especie no identificados lo cual suma un total de 31 muestras vegetales (Tabla 5), mismas que fueron identificadas en el Herbario de la ESPOCH.

\subsubsection{Composición florística de las 5 parcelas de muestreo}

Caranqui et al. [21], menciona que la presencia de un mayor o menor número de especies está influenciado tal vez por el estado de conservación de los páramos o por el grado de intervención. Así en los resultados obtenidos se distingue que la Parcela 3 es la que mayor riqueza posee con 13 familias, 18 géneros y 18 especies, seguida de la Parcela 4 con 13 familias, 16 géneros y 16 especies, como se indica en la Figura 2. Por otro lado la Parcela 1 presenta 8 familias, 10 géneros y 10 especies siendo la parcela con menos diversidad en comparación con la Parcela 3, esto podría deberse a que en el área de estudio hay la presencia de ganado bravo. No obstante la Parcela 2 también presenta 8 familias pero el número de especies es mayor que la Parcela 1 y la Parcela 5 presenta una diversidad media ya que registró 12 familias, 14 géneros y 14 especies. Cabe recalcar que dicha área de estudio se encuentra en un programa de recuperación del páramo por parte de la Regional ya que anteriormente este sitio estaba en proceso de degradación por actividades antrópicas. 


\section{Table 5}

Vegetación registrada.

\begin{tabular}{|c|c|c|c|c|c|}
\hline No. & Familia & Nombre científico & Ge. & Sp. & No. Individuos \\
\hline 1 & Apiaceae & $\begin{array}{l}\text { Daucus montanus Humb et } \\
\text { Bonpl. ex spreng }\end{array}$ & 1 & 1 & 13 \\
\hline 2 & Asteraceae & Lasiocephalus ovatus Schltdl. & 3 & 3 & 11 \\
\hline 3 & & Displostephium sp. & & & 94 \\
\hline 4 & & Werneria & & & 12 \\
\hline 5 & Caprifoliaceae & Valeriana plantaginea Kunth & 1 & 3 & 3 \\
\hline 6 & & $\begin{array}{l}\text { Valeriana rigida (Raiz \& Pav.) } \\
\text { RVdb. }\end{array}$ & & & 9 \\
\hline 7 & & Valeriana microphylla & & & 8 \\
\hline 8 & Caryophyllaceae & Drymania & 1 & 1 & 11 \\
\hline 9 & Dryopteridaceae & Elaphoglossum & 1 & 1 & 8 \\
\hline 10 & Ericaceae & $\begin{array}{l}\text { Disterigma empetrifolium } \\
\text { (Kanth) }\end{array}$ & 2 & 2 & 15 \\
\hline 11 & & Vaccinium cf. floribundum & . & & 45 \\
\hline 12 & Fabaceae & Lupinus sp. & 2 & 2 & 8 \\
\hline 13 & & Trifolium repens $L$. & t & & 18 \\
\hline 14 & Gentianaceae & Gentiana sedifolia Kunth. & 3 & 3 & 16 \\
\hline 15 & & Gentianella sp. & . & & 46 \\
\hline 16 & & Halenia weddeliana Gilg. & & & 3 \\
\hline 17 & Geraniaceae & Geranium sp. & 1 & 2 & 15 \\
\hline 18 & & Geranium laxicaute & & & 91 \\
\hline 19 & Grossulariaceae & Ribes andicola Jancz. & 1 & 1 & 3 \\
\hline 20 & Hypericaceae & Hypericum laricifolium Juss. & 1 & 1 & 7 \\
\hline 21 & Onagraceae & Epilobium denticulatum & 1 & 1 & 8 \\
\hline 22 & Plantaginaceae & Plantago australis Lam. & 1 & 1 & 3 \\
\hline 23 & Poaceae & $\begin{array}{l}\text { Calamagrostis intermedia (J. } \\
\text { Presl) Steud. }\end{array}$ & 2 & 2 & 1153 \\
\hline 24 & & Festuca sp. & & & 21 \\
\hline 25 & Lycopodiaceae & $\begin{array}{l}\text { Huperzia crassa (Humb. \& } \\
\text { Bonpl. Ex Willd) }\end{array}$ & 1 & 1 & 3 \\
\hline 26 & Pteridaceae & Pellaea ternifolia & 1 & 1 & 19 \\
\hline 27 & Ranunculaceae & $\begin{array}{l}\text { Ranunculus praemorsus Humb., } \\
\text { Bonpl. \& Kunth ex DC. }\end{array}$ & 1 & 1 & 5 \\
\hline 28 & Rosaceae & Polylepis racemosa & 2 & 2 & 2 \\
\hline 29 & & $\begin{array}{l}\text { Lachemilia orbiculata (Ruiz \& } \\
\text { Pav.) Rydb }\end{array}$ & t & & 1081 \\
\hline 30 & Liquen & Indeterminada & 1 & 1 & 71 \\
\hline 31 & Musgo & Indeterminada & 1 & 1 & 2497 \\
\hline \multicolumn{2}{|c|}{ TOTAL } & & 26 & 29 & 5299 \\
\hline
\end{tabular}




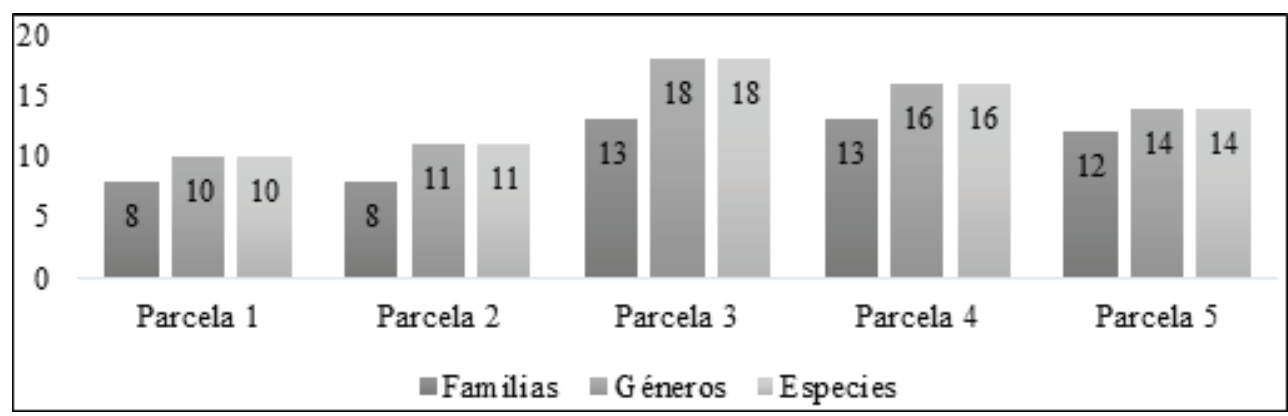

Figure 2

Composición florística por parcela de muestreo.

\section{2. Índice de valor de importancia por especie}

El mayor índice de valor de importancia lo presentó el Musgo (Indeterminada) con un $20,39 \%$, esto se debe a que la especie se encontraba en 4 de las 5 parcelas de muestreo, formando grandes cubiertas esponjosa sobre el suelo del páramo, la especie Calamagrostis intermedia (J. Presl) Steud., ocupó el segundo lugar como especie predominante con $14,11 \%$ debido a que también se encontraba en 4 de las 5 parcelas de muestreo y en tercer lugar tenemos a Lachemilia orbiculata (Ruiz \& Pav.) Rydb como especie dominante de este ecosistema con 12,38\%. Mientras que la especie Halenia weddeliana Gilg. fue la que menor índice de valor de importancia presentó con un $0,37 \%$ esto se debe a que presentó menor cantidad de individuos; seguida por otras especies registradas en este ecosistema (Figura 3). Estos resultados concuerdan con lo mencionado por Hofstede et al. [2], quien menciona que en pendientes fuertes las plantas más tenaces como líquenes y musgos sobreviven.

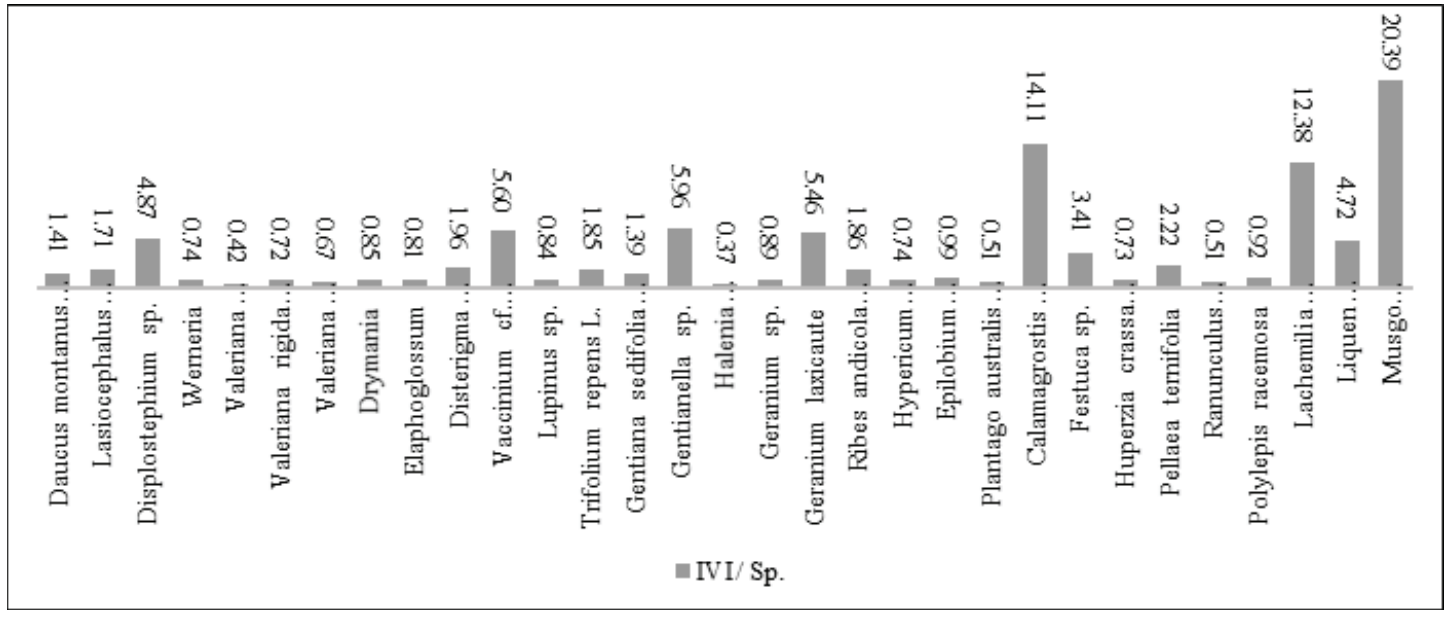

Figure 3

Índice de valor de importancia por especie. 


\section{3. Índice de valor de importancia por familia}

Según la Figura 4, el mayor índice de valor de importancia fue del Musgo (Indeterminada) con un valor de 18,17\%, este se encuentra presente en 4 de las 5 parcelas de muestreo y en 15 de las 20 sub-parcelas de $1 \mathrm{~m}^{2}$, cabe recalcar que esto no disminuye la importancia de las demás familias; las familias Poaceae, Rosaceae, Gentianaceae y Asteraceae también presentan una dominancia en este ecosistema con valores de 15,29\%, 12,15\%, 8,09\% y 7,04\%, respectivamente. Los índices de valor de importancia más bajos fueron para las familias Ranunculaceae, Plantaginaceae, Lycopodiaceae e Hypericaceae con 1,36\%, 1,37\%, 1,37\% y 1,38\% respectivamente. Estos resultados concuerdan con OVACEN [22], quien menciona que la vegetación del páramo es principalmente abierta dominada por vegetales perennes, plantas herbáceas, arbustos y árboles enanos, musgos, líquenes y ciertos pastos. Además se coincide con Bayas [17], que afirma que en los páramos de Ecuador las familias más ricas en géneros y especies son: Asteraceae y Poaceae, seguidas por las familias Ericaceae, Orchidaceae, Cyperaceae, Gentianaceae, Scrophulariaceae, Apiaceae, las cuales ocupan lugares de importancia en otras localidades.

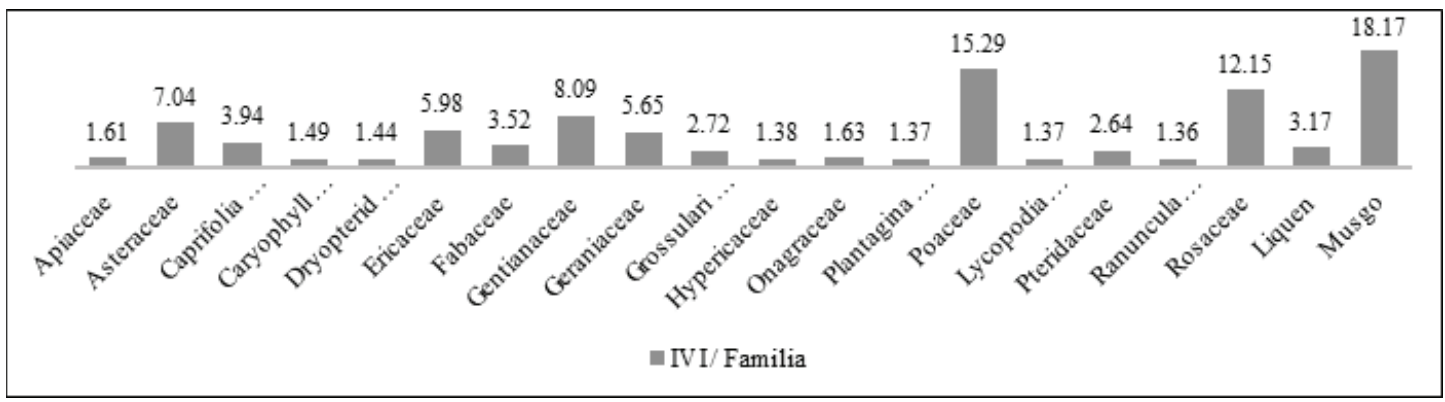

Figure 4

Índice de valor de importancia por familia.

\section{4. Índice de Shannon-Weaver e Índice de Simpson}

Se presentó una diversidad alta está en las parcelas 3 y 4 con valores de 1,00 y 0,96 , respectivamente; lo que nos da a entender que dichas parcelas presentan mayor riqueza dentro de la zona de estudio. Mientras que la parcela 2 arrojó una diversidad baja con un valor de 0,32. Las parcelas 1 y 5 mostraron una diversidad media con valores de 0,73 y 0,70, respectivamente (Tabla 6).

Según el índice de Simpson ninguna de las 5 parcelas registró diversidad alta pero si registraron una diversidad media encontrándose este resultado en las parcelas 3 y 4 con valores muy cercanos de 0,37 y 0,36; mientras que las Parcelas 1, 2 y 5 registraron una diversidad baja con valores de 0,29; 0,11 y 0,25, respectivamente (Tabla 7). Estos resultados coinciden con lo observado en la zona de estudio y reafirma lo mencionado por Caranqui et al. [21], a mayor conservación mayor diversidad. 
Table 6

Índice de Shannon-Weaver por parcela.

\begin{tabular}{|c|c|c|c|}
\hline No. parcela & Valor calculado & Valor referencial & Interpretación \\
\hline P 1 & 0,73 & $0,36-0,75$ & Diversidad media \\
\hline P 2 & 0,32 & $0,00-0,35$ & Diversidad baja \\
\hline P 3 & 1,00 & $0,76-1,00$ & Diversidad alta \\
\hline P 4 & 0,96 & $0,76-1,00$ & Diversidad alta \\
\hline P 5 & 0,70 & $0,36-0,75$ & Diversidad media \\
\hline
\end{tabular}

\section{Table 7}

Índice de Simpson por parcela.

No. parcela
P 1
P 2
P 3
P 4
P 5

Valor calculado
0,29
0,11
0,37
0,36
0,25

Valor referencial
$0,00-0,35$
$0,00-0,35$
$0,36-0,75$
$0,36-0,75$
$0,00-0,35$

Interpretación
Diversidad baja
Diversidad baja
Diversidad media
Diversidad media
Diversidad baja

\section{5. Índice de Sorensen}

El índice de Sorensen muestra la similitud entre las parcelas de muestreo, los resultados mostraron que las cinco parcelas son medianamente similares. La Parcela de muestreo 3 vs Parcela de muestreo 5 presentó una mayor similitud con 0,63 con 10 especies similares, la Parcela de muestreo 1 vs Parcela de muestreo 2 presentó el menor valor de similitud con 0,38 con 4 especies similares (Tabla 8). Estos resultados indican que las especies registradas se pueden encontrar en toda la zona de estudio.

\subsection{Caudal medio mensual}

En Tabla 9, se puede establecer la variación del caudal en el periodo de registro febrero - mayo de 2019, estableciéndose para: La zona 1 valores de $(6,48) ;(6,79) ;(6,87)$ y $(7,59)$ L/s; zona 2 de $(4,52) ;(5,16) ;(4,78)$ y $(4,58) \mathrm{L} / \mathrm{s}$; y la zona 3 de $(6,16) ;(8,45) ;(7,87)$ y $(6,79)$ L/s. Así se determinó un caudal medio mensual de 6,34 L/s. Para [22], los páramos son regiones estratégicas cuya función es la retención de agua y la regulación hídrica durante todo el año.

\subsection{Relación caudal - Precipitación}

El máximo valor se presentó en el mes de marzo con un promedio de $6,80 \mathrm{~L} / \mathrm{s}$, correlacionado con el valor de precipitación que fue de $80,60 \mathrm{~mm}$; mientras que el mes de febrero registró un menor promedio con $5,72 \mathrm{~L} / \mathrm{s}$, lo que coincide con su precipitación que fue de $30,70 \mathrm{~mm}$. Además se puede observar que la curva de tendencia presenta 
Table 8

Índice de Sorensen (similitud entre parcelas).

\begin{tabular}{|c|c|c|c|c|c|}
\hline $\begin{array}{l}\text { No. de } \\
\text { Parcela }\end{array}$ & $\begin{array}{l}\text { Especies } \\
\text { comunes }\end{array}$ & $\begin{array}{l}\text { Valor } \\
\text { calculado \% }\end{array}$ & $\begin{array}{l}\text { Valor } \\
\text { calculado }\end{array}$ & $\begin{array}{l}\text { Valor } \\
\text { referencial }\end{array}$ & Interpretación \\
\hline P 1 vs P 2 & 4 & 38,10 & 0,38 & $0,36-0,70$ & $\begin{array}{l}\text { Medianamente } \\
\text { similares }\end{array}$ \\
\hline $\mathrm{P} 1$ vs $\mathrm{P} 3$ & 7 & 50,00 & 0,50 & $0,36-0,70$ & $\begin{array}{l}\text { Medianamente } \\
\text { similares }\end{array}$ \\
\hline P 1 vs P 4 & 5 & 38,46 & 0,38 & $0,36-0,70$ & $\begin{array}{l}\text { Medianamente } \\
\text { similares }\end{array}$ \\
\hline P 1 vs P 5 & 7 & 58,33 & 0,58 & $0,36-0,70$ & $\begin{array}{l}\text { Medianamente } \\
\text { similares }\end{array}$ \\
\hline P 2 vs P 3 & 8 & 55,17 & 0,55 & $0,36-0,70$ & $\begin{array}{l}\text { Medianamente } \\
\text { similares }\end{array}$ \\
\hline P 2 vs P 4 & 6 & 44,44 & 0,44 & $0,36-0,70$ & $\begin{array}{l}\text { Medianamente } \\
\text { similares }\end{array}$ \\
\hline P 2 vs P 5 & 6 & 48,00 & 0,48 & $0,36-0,70$ & $\begin{array}{l}\text { Medianamente } \\
\text { similares }\end{array}$ \\
\hline P 3 vs P 4 & 10 & 58,82 & 0,59 & $0,36-0,70$ & $\begin{array}{l}\text { Medianamente } \\
\text { similares }\end{array}$ \\
\hline P 3 vs P 5 & 10 & 62,50 & 0,63 & $0,36-0,70$ & $\begin{array}{l}\text { Medianamente } \\
\text { similares }\end{array}$ \\
\hline P 4 vs P 5 & 7 & 46,67 & 0,47 & $0,36-0,70$ & $\begin{array}{l}\text { Medianamente } \\
\text { similares }\end{array}$ \\
\hline
\end{tabular}

Table 9

Registro de caudal mensual y medio mensual.

\begin{tabular}{|c|c|c|c|c|c|c|}
\hline \multirow[t]{2}{*}{ Zona } & \multicolumn{4}{|c|}{ Registro mensual del caudal (L/s) } & \multirow{2}{*}{$\begin{array}{l}\text { Caudal } \\
\text { promedio/zona } \\
\text { (L/s) }\end{array}$} & \multirow{2}{*}{$\begin{array}{l}\text { Caudal medio } \\
\text { mensual (L/s) } \\
6,34\end{array}$} \\
\hline & Febrero & Marzo & Abril & Mayo & & \\
\hline Zona 1 (Vertiente) & 6,48 & 6,79 & 6,87 & 7,59 & 6,93 & \\
\hline Zona 2 (Reservorio 1) & 4,52 & 5,16 & 4,78 & 4,58 & 4,76 & \\
\hline $\begin{array}{l}\text { Zona } 3 \text { (Reservorio de } \\
\text { cloración) }\end{array}$ & 6,16 & 8,45 & 7,87 & 6,79 & 7,32 & \\
\hline $\begin{array}{l}\text { Caudal promedio/mes } \\
\text { (L/s) }\end{array}$ & 5,72 & 6,80 & 6,51 & 6,32 & & \\
\hline
\end{tabular}

una característica polinómica de segundo orden cuya correlación es de 0,82 existiendo una relación positiva, es decir a mayor precipitación existirá mayor caudal (Figura 5).

Es importante relacionar con lo que manifiesta el INAMHI [24], donde en el Ecuador se distinguen dos temporadas: De diciembre a mayo la temporada húmeda, y de junio a noviembre la temporada seca, indicador de la variación del caudal. Además Corredor et al. [24], menciona que la temporalidad del agua disponible depende de patrones climáticos regionales de precipitación y del balance de los componentes del ciclo hidrológico, así como de las características de la vegetación, el suelo y el subsuelo. 


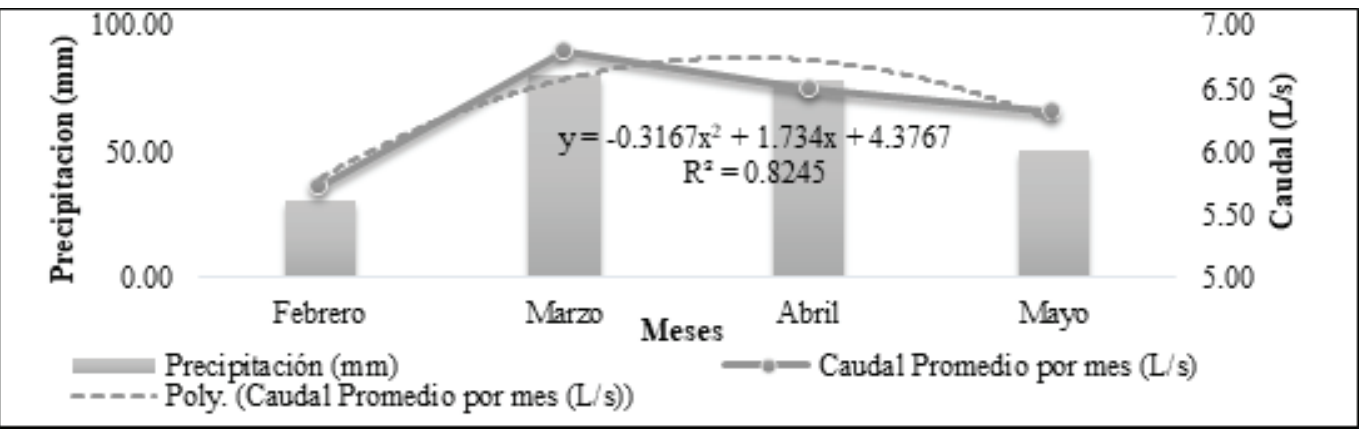

Figure 5

Caudales promedio por mes con respecto a las precipitaciones de febrero a mayo.

\subsection{Caudal promedio por zona}

La zona 3 presentó el mayor caudal con 7,32 L/s esto debido a que en la zona existen corrientes tributarias que ocasionan el aumento de su caudal, mientras que la zona 2 presentó un valor menor con un promedio de 4,76 L/s. Además cabe mencionar que la curva de tendencia presenta una correlación de 0,98 existiendo un grado de correlación (Figura 6). De acuerdo con Bob [25], un caudal debe proporcionar un flujo de al menos 0,125 L/s, es decir que el caudal obtenido en la Regional está dentro de los parámetros permitidos y puede ser distribuido a la población. Sin embargo la demanda de agua, tanto para consumo humano como para actividades productivas, viene en aumento, mientras que la disponibilidad, y la oportunidad del recurso han venido en retroceso [26].

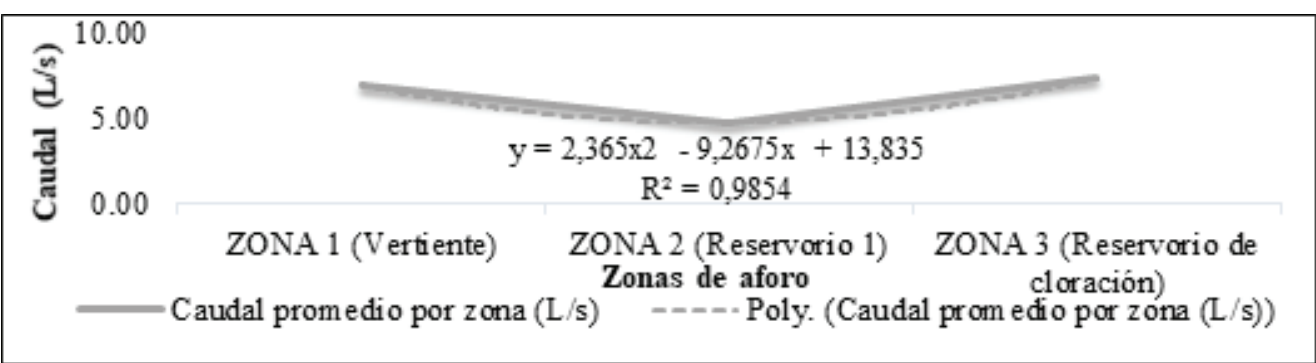

Figure 6

Caudal promedio por zona de aforo.

\subsection{Caudal ecológico}

En función a lo establecido por la legislación ambiental del Ecuador se tomó como referencia el $10 \%$ del caudal mensual [20], obteniéndose los siguientes valores: 0,57 L/s; 0,68 L/s; 0,65 L/s y 0,63 L/s (Figura 7); dándonos un promedio de 0,63 L/s como caudal ecológico. Este valor es el que debe permanecer hasta el momento en la Regional para que el ecosistema páramo mantenga su estructura y equilibrio. 


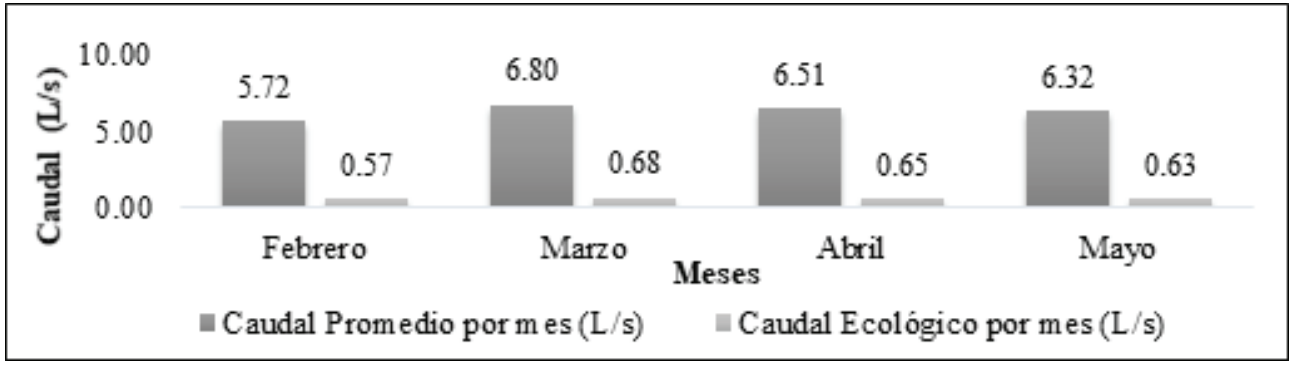

Figure 7

Caudal ecológico mensual de febrero a mayo.

\section{Conclusiones}

El área de estudio registró una diversidad Florística de 5299 individuos agrupados en 18 familias, 26 géneros y 29 especies, también se recolectó musgo y liquen de familia, género y especie no identificados lo cual suma un total de 31 muestras vegetales.

El Musgo (Indeterminada) se encontró en 4 de las 5 parcelas, presentándose con mayor número de individuos en la Parcela 5, esta es una especie con una gran capacidad de retención de agua, además esta especie tiene una gran asociación con el resto de especies que crecen a su alrededor. Las familias que tuvieron mayor índice de valor de importancia (IVI) fueron el Musgo, Poaceae, Rosaceae, Gentianaceae y Asteraceae, lo cual ratifica la riqueza en géneros y especies de estas familias en los páramos del Ecuador.

Según el Índice de Shannon las parcelas que registraron mayor diversidad fueron las parcelas 3 y 4 con valores de 1,00 y 0,96, respectivamente, mientras que el Índice de Simpson indicó que las parcelas 3 y 4 tienen una diversidad media con valores de 0,37 y 0,36 , respectivamente; en el índice de Sorensen las parcelas establecidas en el área de estudio son medianamente similares.

Al aplicar el método volumétrico se obtuvo un caudal promedio mensual de 6,34 $\mathrm{L} / \mathrm{s}$, el cual está dentro de los parámetros permitidos para su distribución, sin embargo la cantidad puede variar no solo por los niveles de precipitación sino por factores antrópicos. El caudal ecológico promedio fue de 0,63 L/s, permitiéndonos conocer la cantidad de agua que debió permanecer en la Regional para mantener el equilibrio del ecosistema páramo. Además es importante saber el valor del caudal ecológico porque sirve de instrumento para la administración y distribución del agua.

\section{Agradecimientos}

A la Regional San José de Chazo-Santa Fé de Galán por haberme dado apertura para la realización de este trabajo de investigación, en especial a los señores operadores y al presidente de la Regional quienes colaboraron con su tiempo para las salidas al páramo. 


\section{References}

[1] Hofstede R, Vásconez S, Cerra M. (Ed.) Vivir en los páramos. Percepciones, vulnerabilidades, capacidades y gobernanza ante el cambio climático [Living in the moors. Perceptions, vulnerabilities, capacities and governance in the face of climate change]. Quito, Ecuador: UICN. 2015. Spanish

[2] Hofstede R, Segarra P, Mena P. Los Páramos del mundo [The paramos of the world]. Proyecto Atlas Mundial de los Páramos. Quito, Ecuador: Global Peatland Initiative/NC-IUCN/EcoCiencia; 2003. Spanish

[3] Hofstede R. Effects of burning and grazing on a Colombian paramo ecosystem [Tesis de doctorado]. Ámsterdam, Holanda: Universidad de Ámsterdam. 1995.

[4] Ramón G. Visiones, usos e intervenciones en los páramos del Ecuador [Visions, uses and interventions in the páramos of Ecuador]. Ecuador: Serie Páramo (Cultura). 2002;(12):43-49. Spanish

[5] Mena P, Hofstede R. Los páramos ecuatorianos [Equatorial paramos]. Revista Botánica Económica de los Andes Centrales. La Paz, Bolivia. 2006. p 94-95. Spanish

[6] Jaramillo A. Plan de manejo de los recursos naturales de la Regional San José de Chazo - Santa Fe de Galán. Estrategia de Conservación articulada al proceso de desarrollo local [Natural resources management plan of the San José de Chazo - Santa Fe de Galán Regional Region. Conservation Strategy articulated to the local development process]. Riobamba, Ecuador; 2014. Spanish

[7] Ministerio del Ambiente (MAE). Sistema de clasificación de ecosistemas de Ecuador Continental [Ecosystem classification system of Continental Ecuador,Internet]. 2014 [Recuperado el 2018 Oct 19]. Disponible de: http://app.sni.gob.ec/snilink/sni/PDOT/NIVEL\{\%\}20NACIONAL/MAE/ECOSISTEMAS/ DOCUMENTOS/Sistema.pdf. Spanish

[8] Pauli H, Gottfried M, Lamprecht A, Niessner S, et al. Manual para el trabajo de campo del proyecto Gloria [Manual for the fieldwork of the Gloria project,Internet]. Aproximación al estudio de las cimas. Métodos básicos, complementarios y adicionales. $5^{a}$ edición. GLORIA - Viena, Austria: Coordinación, Academia Austriaca de Ciencias y Universidad de Recursos Naturales y Ciencias de la Vida. Edición en español a cargo de Benito JL, Villar L, Jaca, España. 2015 [Recuperado el 2018 Oct] Disponible de: https://www.researchgate.net/profile/Jose_Luis_Benito_Alonso/publication/282567915_Manual_par a_el_trabajo_de_campo_del_proyecto_GLORIA_Aproximacion_al_estudio_de_las_cimas_Metodos _basico_complementarios_y_adicionales_5_edicion/links/5615380308ae4ce3cc6526b3/Manual-par a-el-trabajo-de-campo-del-proyecto-GLORIA-Aproximacion-al-estudio-de-las-cimas-Metodos-basic o-complementarios-y-adicionales-5-edicion.pdf

[9] Peralta J. Determinación del caudal y calidad de agua mediante bioindicadores en los páramos de la comunidad el calvario, ubicado en el cantón Tisaleo, provincia de Tungurahua [Determination of water flow and quality using bioindicators in the moors of the El Calvario community, located in the Tisaleo canton, Tungurahua province] [Tesis de grado. Ingeniera Forestal]. Riobamba: ESPOCH; 2019. Spanish

[10] Sonco R. Estudio de la diversidad alfa $(\alpha)$ y beta $(\beta)$ en tres localidades de un bosque montano en la región de Madidi, La Paz-Bolivia [Study of alpha $(\alpha)$ and beta $(\beta)$ diversity in three localities of a montane forest in the Madidi region, La Paz-Bolivia,Tesis de Grado. Ingeniero Agrónomo]. La Paz: Universidad Mayor de San Andrés; 2013. Spanish

[11] Campo A, Duval V. Diversidad y valor de importancia para la conservación de la vegetación natural [Diversity and value of importance for the conservation of natural vegetation]. Parque Nacional Lihué Calel (Argentina). Madrid, España: Revista Anales de Geografía de la Universidad Complutense. 2013;(34):32. Spanish

[12] Aguirre (2006)

[13] Pujos L. Diversidad florística a diferente altitud en el ecosistema páramo de tres comunidades de la organización de segundo grado unión de organizaciones del pueblo Chibuleo [Floristic diversity at different altitudes in the páramo ecosystem of three communities of the second degree organization union of organizations of the Chibuleo people,Tesis de grado. Ingeniero Forestal]. Riobamba: ESPOCH; 2013. Spanish

[14] Smith \& Smith (2001).

[15] Valencia C. Índices de diversidad [Diversity indexes,Internet]. 2013 [Recuperado el 2019 Oct 19]. Disponible en: https://es.slideshare.net/ccvalenciac/indices-de-diversidad-ecosistmica. Spanish Ordoñez et al. (2009).

[16] Bayas D. Diversidad florística a diferente altitud en el ecosistema páramo del cantón Tisaleo provincia de Tungurahua [Floristic diversity at different altitudes in the páramo ecosystem of the Tisaleo canton, Tungurahua province,Tesis de grado Ingeniero Forestal]. Escuela Superior Politécnica de Chimborazo. Riobamba: ESPOCH; 2015. Spanish

[17] Lozano A, Sánchez J. Evaluación experimental de una ecuación empírica para la caída de presión en flujo gaseoso [Experimental evaluation of an empirical equation for pressure drop in gas flow,Internet]. 2017 [Recuperado 17 de marzo, del 2018]. Disponible en: http://repositorio.uncp.edu.pe/bitstream/ handle/UNCP/3786/Lozano\{\%\}20Povis\{\%\}20Sanchez\{\%\}200choa.pdf?sequence=1\&isAllowed=y 
[18] Bello M, Pino M. Medición de presión y caudal [Pressure and flow measurement,Internet]. 2000 [Recuperado 2018 May 5]. Disponsible de: http://biblioteca.inia.cl/medios/biblioteca/boletines/ NR25635.pdf. Spanish

[19] López J. Caudal ecológico en Ecuador [Ecological flow in Ecuador,Internet]. IAGUA; 2018 [Recuperado el 2018 Oct 19]. Disponible en: https://www.iagua.es/blogs/juan-calles-lopez/ caudal-ecologico-ecuador. Spanish

[20] Caranqui J, Lozano P, Reyes J. Composición y diversidad florística de los páramos en la Reserva de Producción de Fauna Chimborazo, Ecuador [Composition and floristic diversity of the páramos in the Chimborazo Fauna Production Reserve, Ecuador]. Quito, Ecuador: Enfoque UTE. 2016;(7):33-42. Spanish

[21] OVACEN. El Páramo [The paramo,Internet]. 2016 [Recuperado el 2018 Oct 19] Disponible en: https: //ecosistemas.ovacen.com/bioma/paramo/. Spanish

[22] Instituto Nacional de Meteorología e Hidrología (INAMHI). Tipos de clima Ecuador [Climate types Ecuador,Internet]. 2017 [Recuperado el 2018 Oct 19]. Disponible en: http://www.serviciometeorologico. gob.ec/. Spanish

[23] Corredor E, Fonseca J,Páez E. Los servicios ecosistémicos de regulación: tendencias e impacto en el bienestar humano [Regulating ecosystem services: trends and impact on human well-being]. Colombia: Revista de Investigación Agraria y Ambiental. 2012;(3):78-80. Spanish

[24] Bob R. Cantidad mínima de agua necesaria para uso doméstico [Minimum amount of water required for domestic use,Internet]. 2000 [Recuperado el 2018 Oct 19]. Disponsible en: http://www.disasterinfo. net/Agua/pdf/9-UsoDomestico.pdf. Spanish

[25] Díaz S. Biodiversity regulation of ecosystem services, en R. Hassan, R. Scholes y N. Ash (eds.), Ecosystems and hu-human well-being: Current state and trends, (I) Findings of the Condition and Trends Working Group of the Millennium Ecosystem Assessment. Washington, D.C., Estados Unidos: Island Press. 2006. 